\title{
Epigenetic Repression of Matrix Metalloproteinases in Myofibroblastic Hepatic Stellate Cells through Histone Deacetylases 4
}

\author{
Implication in Tissue Fibrosis
}

\author{
Lan Qin* and Yuan-Ping Han ${ }^{\star \dagger \ddagger \S ~}$ \\ From the Graduate Program of Systems Biology and Disease, ${ }^{*}$ the \\ Departments of Surgery, ${ }^{\dagger}$ and Pathology, ${ }^{*}$ the Keck School of \\ Medicine, University of Southern California, Los Angeles, \\ California; and the Center for Growth, Metabolism and Aging \\ Research, ${ }^{\S}$ College of Life Sciences, Sichuan University, Chengdu, \\ Sichuan, China
}

\begin{abstract}
Matrix metalloproteinases (MMPs), which are highly expressed in acute injury, are progressively repressed or silenced in fibrotic liver, favoring extracellular matrix accumulation, while the underlying mechanism is largely unknown. Similarly, normal/ quiescent hepatic stellate cells (HSCs) express high levels of MMPs in response to injury signals, such as interleukin-1. After transdifferentiation, the myofibroblastic HSCs are incapable of expressing many MMPs; however, the major signaling pathways required for MMP expression are intact, indicating that repression is at the level of the chromatin. Indeed, both the MMP9 and MMP13 genes are inaccessible to transcription factors and RNA polymerase II, in association with impaired histone acetylation in their promoters. In accordance with impaired histone acetylation at the cellular level, histone deacetylase- 4 is accumulated during HSC transdifferentiation. Furthermore, ectopic expression of histone deacetylase- 4 in quiescent HSCs results in repression of MMP promoter activities as well as endogenous MMP9 protein expression. Thus, our findings suggest that a histone deacetylase-4-dependent mechanism underlies the epigenetic silencing of MMP genes during tissue fibrogenesis. (Am J Pathol 2010, 177:1915-1928; DOI: 10.2353/ajpath.2010.100011)
\end{abstract}

Matrix metalloproteinases (MMPs) are a family of endopeptidases, which are expressed in embryonic devel- opment and remain expressed in actively growing adult tissues. ${ }^{1}$ In developed tissues, most MMP genes are dormant, but they can be re-activated in the cases of tissue injury, infection, and tumor growth. Chronic infection and injury usually result in tissue fibrosis, characterized by transdifferentiation of normal cells into myofibroblasts, accumulation of extracellular matrix (ECM), and suppression of MMPs. ${ }^{2,3}$ Liver fibrosis is mostly mediated by hepatic stellate cells (HSCs), the pericytes in liver, 4,5 which lodge underneath sinusoidal endothelia in looselypacked ECM, a unique region called "the space of Disse."6,7 In normal liver, quiescent HSCs produce most hepatic ECM and store most retinoids. In response to inflammatory cytokines such as interleukin-1 (IL-1), HSCs express many MMPs in acute hepatic damage. ${ }^{8}$ It is generally accepted that MMPs function in tissue injury and wound healing by releasing growth factors, promoting cell migration, and cellular differentiation. ${ }^{9}$ As the consequence of chronic liver injury such as hepatic viral infection and alcohol overconsumption, quiescent HSCs undergo progressive transdifferentiation, called "activation," through which they are converted to myofibroblastlike cells as marked by increased expression of $\alpha$-smooth muscle actin ( $\alpha$-SMA) and interstitial ECM fibers as well as enhanced contractibility. ${ }^{10-14}$ Notably, the end-point myofibroblastic HSCs are incapable of expressing most MMPs (except MMP2) even under inflammatory stimulation, ${ }^{15}$ a phenomenon that is crucial for accumulation of

Supported by NIH funding (R01AR051558 and R01DK069418), and the Wright Foundation (Y.-P.H.) The Southern California Research Center for Alcoholic Liver and Pancreatic Diseases and Cirrhosis is supported by $\mathrm{NIH}$ grant R24AA12885. The USC Research Center for Liver Diseases microscopy subcore is supported by NIH grant P30 DK 048522.

Accepted for publication June 17, 2010.

Supplemental material for this article can be found on http://ajp. amjpathol.org.

Address reprint requests to Yuan-Ping Han, Ph.D., 2011 Zonal Avenue HMR 813, Los Angeles, CA 90033. E-mail: yhan@surgery.usc.edu. 
ECM in fibrotic tissues. However, little progress has been made in uncovering the mechanism underlying MMP repression in tissue fibrosis.

Mesenchymal cells including HSCs are restricted to express MMPs by demanding simultaneous stimuli of ECM and the injury signals such as inflammatory cytokines. ${ }^{16,17}$ For MMP expression, HSCs must be at "quiescent state" and with simultaneous exposure to injury signals such as IL-1; three dimensional (3D) ECM can further boost the MMP expression. Such tight requirement of both extracellular cues and intracellular settings underscores the critical roles of MMPs in liver tissues, and such notion has been demonstrated by the findings that excessive amounts of MMPs in liver are lethal. ${ }^{18}$ Many "injury-type" MMPs, such as MMP9 and MMP13 are induced by stress signals, and are mediated by JNK and IKK signaling pathways. ${ }^{19,20}$

Transcription in eukaryotic cells begins on binding of signal-induced transcription factors to the cis-elements on gene promoters, and is then followed by recruitment of transcription co-activators, including histone acetyltransferases (HATs) that add acetyl groups to histone tails and consequently result in unpacking of DNA from nucleosome and loading of the transcription apparatus. $^{21,22}$ The extent of histone acetylation is a benchmark of active genes in transcription. ${ }^{23,24}$ Conversely, histone deacetylation, the reverse process which is mediated by histone deacetylases (HDACs), is associated with gene repression and heterochromatin. ${ }^{25,26,27}$ Members of HDAC superfamily have been classified according to their sequence feature and domain organization. ${ }^{28}$ Class I HDACs, including HDAC-1, -2 , and -3 , are ubiquitously expressed and reside in nucleus, ${ }^{29}$ while class II HDACs (HDAC4, $-5,-6,-7,-9$ and -10$)$ are cell type specific and able to shuttle between cytoplasm and nucleus depending on their phosphorylation states. ${ }^{30}$ To date, whether and how HDACs are involved in MMP gene silencing during tissue fibrosis is mostly unknown.

To understand the mechanism of MMP silencing during HSCs transdifferentiation, we used both in vivo and in vitro models. We first confirmed the concept of MMP silencing in a mouse model. Then, we recapitulated the key features of MMP silencing in vitro using rat primary HSCs. We examined whether key signaling pathways controlling MMP expression are defective during HSC transdifferentiation. We discovered that the impaired transcription factor recruitment to the MMP genes correlates to the decreased histone acetylation in myofibroblastic HSCs. Moreover, accompanying transdifferentiation, HDAC4 is progressively accumulated in line with histone deacetylation and MMP repression. Finally, ectopic expression of HDAC4 in quiescent HSCs could lead to suppression of the MMP promoter activities and repression of endogenous MMP9 expression. Taken together, silencing of MMP genes is likely mediated by epigenetic switch, in part, through HDAC4 upregulation during HSC transdifferentiation in liver fibrosis.

\section{Materials and Methods}

\section{Animal Model}

Mice (FVB, from the Jackson Laboratory, Bar Harbor) were divided into three groups: (1) control, (2) acute injury, and (3) fibrosis $(n=6)$. Saline was given to groups 1 and 2, while thioacetamide (TAA) at $0.2 \mathrm{mg} / \mathrm{g}$ body weight was given to group 3 by repeating injection (intraperitoneal), twice a week for 8 weeks. Twenty-four hours before sacrifice of the mice, the group 2 and 3 mice were subjected to a final TAA injection. There was no mortality associated with the injection. Rats (Wistar) were injected with carbon tetrachloride at $0.5 \mathrm{ml} / \mathrm{kg}$ body weight or mineral oil as control, and then sacrificed on day 4. Livers were collected for zymography and immunohistological studies. All animals received humane care in compliance with the institution's guidelines for the care and use of laboratory animals in research.

\section{Isolation of Rat HSCs}

Protocols for isolation of rat HSCs have been previously described. . $^{31,32}$ HSCs were isolated from retired normal male Wistar rats. Both the purity and viability of HSCs always exceed 95\% (Provided by the Southern California Research Center for ALPD and Cirrhosis).

\section{Cell Culture}

Primary rat HSCs were cultured on plastic in Dulbecco's modified Eagle's medium with 10\% fetal bovine serum and 100 units/ml penicillin-streptomycin for 2 days before experiments. For 3D ECM culture, cells were trypsinized and embedded in collagen as described previously. ${ }^{15}$ Myofibroblastic HSCs were prepared by culturing the primary cells on plastic for up to 10 days in Dulbecco's modified Eagle's medium with 10\% fetal bovine serum, wherein medium was changed every 3 days.

\section{Western Blot}

To collect whole cell lysates, after PBS wash, $1 \times$ reducing sample buffer $(62.5 \mathrm{mmol} / \mathrm{L}$ Tris- $\mathrm{HCl}, \mathrm{pH} 6.8,2 \%$ SDS, $10 \%$ glycerol, $0.0025 \%$ bromophenol blue and 0.1 $\mathrm{mol} / \mathrm{L}$ dithiothreitol) was added to cells cultured on plastic. For cells cultured in collagen, the gel lattices were taken into $1.5-\mathrm{ml}$ tube and centrifuged at $6000 \mathrm{rpm}$ at $4^{\circ} \mathrm{C}$ for 5 minutes, and the pellet was dissolved in $1 \times$ reducing sample buffer. For conditioned medium, equal volume was mixed with $2 \times$ reducing sample buffer. After 5 minutes of boiling, samples were centrifuged at 14,000 rpm for 10 minutes at $4^{\circ} \mathrm{C}$. The supernatant was transferred to a fresh tube and total protein concentration was measured using Bio-Rad protein assay according to the manufacture's instruction. Twenty $\mu \mathrm{g}$ protein of each sample was resolved by SDS-polyacrylamide gel electrophoresis, and then transferred to Immobilon-P membrane (Millipore IPVH00010). After 3 hour blocking in 5\% nonfat milk or bovine serum albumin according to manufactur- 
er's datasheet, the membrane was incubated with 1 $\mu \mathrm{g} / \mathrm{ml}$ primary antibody overnight at $4^{\circ} \mathrm{C}$. After that, the membrane was washed and then further incubated with horseradish peroxidase-conjugated secondary antibodies from Santa Cruz Biotechnology (sc-2020, sc-2005, sc-2004), and developed by Super Signal West Femto Chemiluminescent substrate (34096, Pierce). AntiMMP13 (MAB 13426) and glyceraldehyde-3-phosphate dehydrogenase (GAPDH; MAB 374) antibodies were purchased from Millipore. Antibodies for $I_{\kappa} \mathrm{B} \alpha(9242)$, p-JNK (9255), and JNK (9258), as well as HDAC1, 2, 3, 4, 5, 7 (9928), histone H3 (9715) and histone H4 (2592) were purchased from Cell Signaling, while anti-ERK (sc-93) and p-ERK (sc-7383) were from Santa Cruz Biotechnology. Antibody for $\beta$-actin (A2228) was from Sigma. Acetyl-histone $\mathrm{H} 4$ antibodies were purchased from Millipore (17-211).

\section{Zymography}

Conditioned medium, in sample buffer without dithiothreitol, was resolved by $10 \%$ SDS-polyacrylamide gel electrophoresis containing $0.1 \%(w / v)$ gelatin (G9382, Sigma). To enrich gelatinases for zymography analysis, mouse liver tissues $(50 \mathrm{mg})$ were homogenized into 1.0 $\mathrm{ml}$ cold NT buffer (50 mmol/L Tris-HCl pH7.5, $100 \mathrm{mmol} / \mathrm{L}$ $\mathrm{NaCl}$ ) containing $1 \%$ Triton $\mathrm{X}-100$. After 20 minutes centrifugation at $12,000 \mathrm{rpm}$ at $4^{\circ} \mathrm{C}$, the supernatant was taken to measure total protein concentration by Biorad protein assay (500-0006) and samples with equal total protein amount were loaded for analysis. After electrophoresis, the gel was washed in $2 \%$ Triton X-100 for 30 minutes and then incubated with developing buffer (5 $\mathrm{mmol} / \mathrm{L} \mathrm{CaCl}{ }_{2}, 150 \mathrm{mmol} / \mathrm{L} \mathrm{NaCl}, 50 \mathrm{mmol} / \mathrm{L}$ Tris, $\mathrm{pH}$ 7.5) for 16 hours at $37^{\circ} \mathrm{C}$ and visualized by Coomassie Blue R-250 staining.

\section{Plasmid Construction}

Rat genomic DNA was extracted from primary HSCs. Briefly, cells were collected into TE buffer $(200 \mathrm{mmol} / \mathrm{L}$ Tris- $\mathrm{HCl}$ pH8.0, $100 \mathrm{mmol} / \mathrm{L}$ EDTA). Washed with TE buffer, cells were treated with $0.4 \mathrm{mg} / \mathrm{ml}$ proteinase $\mathrm{K}$ and $1 \%$ SDS in TE buffer overnight. Then, DNA was purified by phenol/chloroform extraction and isopropyl alcohol precipitation. PCR was performed to construct the promoter regions of MMP9 and MMP13 using primers listed in Table 1. For the promoter of MMP9, the primers include a Kpnl site at the $5^{\prime}$ end and Bglll site at the other end; for the promoter of MMP13, the primers have a Kpnl site at the $5^{\prime}$ end and a Smal site at the other. After restriction enzyme digestion (Kpnl and Bglll for MMP9, Kpnl and
Smal for MMP13), the PCR products were cloned into pGL3-basic vector harboring a firefly luciferase (Promega) gene as reporter plasmids, namely, p1300MMP9luc, and p1800MMP13-luc. To express HDAC4, cDNA encoding mouse HDAC4 was purchased from Open Biosystems. After digestion with EcoRI and Notl, the insert was cloned into pcDNA3 (Invitrogen), prepared by those two restriction enzymes. The inserts were confirmed by DNA sequencing performed by the DNA core facility in Norris Cancer Center at University of Southern California.

\section{Transfection and Luciferase Assay}

Transfection of primary HSCs was carried out with Lipofectamine 2000 (Invitrogen) according to manufacturer's manual. Briefly, HSCs were subcultured into 24-well plate at the density of 0.1 million cells per well. After 6 hours, transfection was performed using $1 \mu \mathrm{g}$ of reporter plasmid, $2 \mu \mathrm{g}$ of HDAC4/pcDNA3 together with $10 \mathrm{ng}$ of phRL (CMV promoter driven renilla luciferase). After 18 hours, the cells were treated with or without IL-1 for an additional 18 hours. Conditioned medium was collected for zymography analysis. Cells were collected for luciferase assay, using dual luciferase assay system (Promega) according to the manufacturer's suggestion.

\section{Chromatin Immunoprecipitation Assay}

Chromatin immunoprecipitation (ChIP) assay was performed as previously described, with modification. ${ }^{33}$ After stimulation with cytokines, HSCs were cross-linked by $1 \%$ formaldehyde for 10 minutes at room temperature. Then cells were washed with ice cold PBS twice, and scraped into ice cold PBS containing protease inhibitor cocktails (S8820, Sigma) and collected by 10 minutes centrifugation at $3000 \mathrm{rpm}$ at $4{ }^{\circ} \mathrm{C}$. Cell pellets were resuspended in cell lysis buffer $(5 \mathrm{mmol} / \mathrm{L}$ Pipes/potassium $\mathrm{pH} 8.0,85 \mathrm{mmol} / \mathrm{L} \mathrm{KCl}, 0.5 \%$ CA-630 plus protease inhibitor cocktails) and incubated on ice for 10 minutes with occasional vortex. After a 10-minute spin at $5000 \mathrm{rpm}$, the nuclei pellets were lysed in SDS lysis buffer $(50 \mathrm{mmol} / \mathrm{L}$ Tris $\mathrm{pH}$ 8.0, $10 \mathrm{mmol} / \mathrm{L}$ EDTA, $1 \%$ SDS plus protease inhibitors), followed by a 10-minute incubation on ice. Sonication was then carried out to get chromatin fragments between 200 to1,000 bp. After that, the mixture was 10 -fold diluted with radioimmunoprecipitation assayChIP buffer [10 mmol/L Tris-HCl, pH 7.5, $140 \mathrm{mmol} / \mathrm{L}$ $\mathrm{NaCl}, 1 \mathrm{mmol} / \mathrm{L}$ EDTA, $0.5 \mathrm{mmol} / \mathrm{L}$ EGTA, 1\% (v/v) Triton X-100, $0.1 \%(\mathrm{w} / \mathrm{v})$ SDS, $0.1 \%(\mathrm{w} / \mathrm{v})$, Na-deoxycholate, protease inhibitor mix], and centrifuged at $14,000 \mathrm{rpm}$ for 10 minutes at $4^{\circ} \mathrm{C}$. The supernatant containing chromatin preparation was transferred to a new tube and aliquoted

Table 1. Primers for Cloning of MMP9 and MMP13 Promoters from Rat Genomic DNA

\begin{tabular}{cl}
\hline Name & \multicolumn{1}{c}{ Sequence } \\
\hline p1300MMP9-luc & $5^{\prime}$-GGGGTACCTGTGGCTTGAAGGCGAAATGC-3' \\
p1800MMP13-luc & $5^{\prime}$-GAAGATCTGGTGAGAACCGAGCTTCTGGG-3' \\
& $5^{\prime}$-GGGGTACCTGGCACAAGCTGTAATCCTAGCACT-3' \\
& $5^{\prime}-$ TCCCCCGGGCTCAACAAGAAGAAGGTGGCCAGA-3' $^{\prime}$ \\
\hline
\end{tabular}


Table 2. Primers for ChIP Assay

\begin{tabular}{cc}
\hline \multicolumn{1}{c}{ Name } & Sequence \\
\hline Primer set for & 5'-AAGGAGTCAGCCTGCTGGG-3' \\
MMP9 distal & 5'-CCCACACTGTAGGTTCTATCCTCT-3' \\
site (1) & \\
Primer set for & 5'-TGAGTCAGCGTAAGCCTGGA-3' \\
MMP9 proximal & 5'-GGTGAAGCAGAATTGCGGAGGTT-3' \\
site (2) & \\
Primer set for & 5'-AAGTCCCAAATGGTCTCGGTCTGA-3' \\
MMP13 & $5^{\prime}$-AAACGGTTCTGACAAAGGCTGCTG-3' \\
proximal & \\
site (1) & \\
Primer set for & 5'-ATCCTGTCAGCTGTCTGCGATCT-3' \\
MMP13 middle & 5'-TGGACAGCCAGCCTTAAGGAAATG-3' \\
site (2) & \\
Primer set for & 5'-CTGCCACAAACCACACGTACGAAA-3' \\
MMP13 distal & $5^{\prime}$-CTTCCCAGGGCAAGCATTCTCTAT-3' \\
site (3) & \\
\hline
\end{tabular}

for subsequent experiment. For each assay, the chromatin preparation was first incubated with $1.2 \mu \mathrm{g}$ of primary antibodies overnight on rotator at $4^{\circ} \mathrm{C}$. And then $10 \mu \mathrm{l}$ of radioimmunoprecipitation assay buffer pre-washed Dynabeads protein-A (Invitrogen) was added and incubated for another hour. After that, the beads were sequentially washed with radioimmunoprecipitation assayChIP buffer for three times and then TE buffer $(10 \mathrm{mmol} / \mathrm{L}$ Tris- $\mathrm{HCl}, \mathrm{pH}$ 8.0, $10 \mathrm{mmol} / \mathrm{L}$ EDTA) twice. Pulled down DNA was eluted and reverse cross-linked by incubation with elution buffer [20 mmol/L Tris-HCl, $\mathrm{pH}$ 7.5, $5 \mathrm{mmol} / \mathrm{L}$ EDTA, $50 \mathrm{mmol} / \mathrm{L} \mathrm{NaCl}, 1 \%$ (w/v) SDS, $50 \mu \mathrm{g} / \mathrm{ml}$ proteinase $\mathrm{K}$ ] twice at $68^{\circ} \mathrm{C}$ for a total of 2 hours. DNA was then purified by phenol-chloroform extraction and ethanol precipitation, and amplified by quantitative real-time (qRT) PCR. Antibodies for acetyl-histone H3 (06-599) and H4 (06-598) were from Millipore. Antibodies for C-Jun (sc45) were from Santa Cruz Technology. Anti RNA polymerase II CTD repeat YSPTSPS (phospho S5) (ab5131) was purchased from Abcam. For relative quantitative ChIP assay, the thermal cycle was set at $94^{\circ} \mathrm{C} 10$ minutes, followed by 50 cycles of 20 seconds at $94^{\circ} \mathrm{C}$ and 1 minute at $60^{\circ} \mathrm{C}$. The final result was represented as

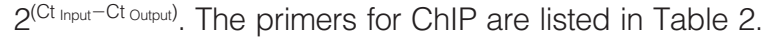

\section{qRT-PCR Analysis}

RNA was extracted from mouse livers using RNAqueous 96 (Ambion) and from HSCs using Trizol Reagent (Invitrogen). Reverse transcription was carried out with random primer and M-MLV (Invitrogen) according to the manual. Real-time PCR was performed using SYBR master mix (Applied Biosystems) and gene specific primers (Table 3). The reaction was carried out in 384-well plate with $A B I$ 7900 DNA detection system at USC Research Center for Liver Diseases. Each reaction was performed in triplicate and $5 \mathrm{ng}$ of cDNA was used in a $10 \mu \mathrm{l}$ system for each well. To measure mRNA from the reverse transcription, the reaction was carried out at $94^{\circ} \mathrm{C}$ for 10 minutes, followed by 40 cycles of 10 seconds at $94^{\circ} \mathrm{C}$ and 1 minute at $60^{\circ} \mathrm{C}$. The final result of gene transcription was
Table 3. Primers for Quantitative Real-Time PCR

\begin{tabular}{|c|c|}
\hline Name & Sequence \\
\hline Mouse MMP-9 & $\begin{array}{l}\text { 5'-CGTGTCTGGAGATTCGACTTGA-3' } \\
\text { 5'-TGGAAGATGTCGTGTGAGTTCC-3' }\end{array}$ \\
\hline Mouse & 5'-AGGTGGTACACTAGCCCATGCTTT-3' \\
\hline $\begin{array}{l}\text { Mouse } \\
\text { MMP-14 }\end{array}$ & $\begin{array}{l}\text { 5'-ATCTCACAGCTCGGTGTGTGTTCA-3' } \\
\text { 5'-AAGGTCAGAGGGTCTTGCCTTCAA-3' }\end{array}$ \\
\hline $\begin{array}{l}\text { Mouse type I } \\
\text { collagen }\end{array}$ & $\begin{array}{l}5^{\prime}-\text { GCATGGCCAAGAAGACATCC-3' } \\
5^{\prime}-\text {-CCTCGGGTTTCCACGTCTC-3' }\end{array}$ \\
\hline $\begin{array}{l}\text { Mouse } \\
\text { GAPDH }\end{array}$ & $\begin{array}{l}5^{\prime}-\text { GCACAGTCAAGGCCGAGAAT-3' } \\
5^{\prime} \text {-GCCTTCTCCATGGTGGTGAA-3' }\end{array}$ \\
\hline Rat MMP-9 & $\begin{array}{l}\text { 5'-CAGACCAAGGGTACAGCCTGTT-3' } \\
5^{\prime} \text {-AGCGCATGGCCGAACTC-3' }\end{array}$ \\
\hline Rat MMP-12 & $\begin{array}{l}\text { 5'-TCTATGGAGCCCCAGTGAAA-3' } \\
5^{\prime} \text {-GACACACAGTTGATGGTGGACTTC-3' }\end{array}$ \\
\hline Rat MMP-13 & $\begin{array}{l}5^{\prime}-\text { GCCCTATCCCTTGATGCCATT-3' } \\
5^{\prime}-\text { ACAGTTCAGGCTCAACCTG-3' }\end{array}$ \\
\hline Rat MMP-14 & $\begin{array}{l}5^{\prime} \text {-GCCCAACATCTGTGATGGGAACTT-3' } \\
5^{\prime} \text {-TTATTCCTCACCCGCCAGAACCAT-3' }\end{array}$ \\
\hline Rat GAPDH & $\begin{array}{l}\text { 5'-CCTGGAGAAACCTGCCAAGTAT-3' } \\
5^{\prime}-\text { CTCGGCCGCCTGCTT- } 3^{\prime}\end{array}$ \\
\hline
\end{tabular}

calculated as $2^{\left(\mathrm{Cl}_{\mathrm{GAPH}}-\mathrm{Ct} \text { Gene) }\right.}$. The primers for qRT-PCR are listed in Table 2.

\section{Immunofluorescent Staining}

Liver tissues were snap frozen in liquid nitrogen and embedded in optimal cutting temperature compound. Sections were fixed in ice-cold acetone for 10 minutes. After blocking with $5 \%$ donkey serum in PBS, specimens were further incubated overnight with $4 \mu \mathrm{g} / \mathrm{ml}$ of the primary antibody (anti-MMP9, sc-6841, Santa Cruz Biotechnology), anti-MMP13 (AB8120, Millipore), and antidesmin (sc-7559, Santa Cruz Biotechnology and ab8592, Abcam). Cy3 conjugated rabbit anti-goat IgG (C2821, Sigma) and fluorescein isothiocyanate-conjugated goat anti-mouse IgG (F2057) were selectively used for visualization. For immunofluorescent staining, HSCs were fixed with $4 \%$ formaldehyde in PBS for 10 minutes at room temperature. After three washes with PBS, cells were permeabilized with $0.1 \%$ Triton X-100 in PBS for 10 minutes. Then the cells were blocked with $5 \%$ donkey serum in PBS and further incubated with $4 \mu \mathrm{g} / \mathrm{ml}$ of primary antibody overnight. Then fluorescence conjugated secondary antibodies (A-11057, A-21206, Invitrogen) were applied. To stain F-actin, tetramethylrhodamine B isothiocyanate-conjugated phalloidin (P1951, Sigma) at $0.1 \mu \mathrm{g} / \mathrm{ml}$ was applied. Nuclei were visualized by 4,6-diamidino-2-phenylindole at $0.1 \mu \mathrm{g} / \mathrm{ml}$. Pictures were taken with a Zeiss confocal microscope in the USC Research Center for Liver Diseases or a Nikon TU2500Eclipse in this lab.

\section{H\&E and Sirius Red Staining of Liver Sections}

Paraffin sections were deparaffinized and rehydrated by sequential treatments of xylene, $100 \%$ and $95 \%, 80 \%$ ethanol, followed by deionized water. The slides were incubated with Hematoxylin followed by rinsing with water and quick dips into acid ethanol to destain. For eosin 
staining, the slides were incubated shortly with eosin, followed by $95 \%$, 100\% ethanol and xylene. For Sirius Red staining, the sections were deparaffinized and then stained by Fast Green and Sirius Red sequentially. Lastly, the sections were dehydrated by ethanol and xylene treatments before mounting.

\section{Statistical Analysis}

Numerical data were expressed as means +/- SD The significance of the differences between two groups was assessed using Student's $t$-test. A $P$ value of less than 0.05 was considered statistically significant.

\section{Results}

\section{Liver Fibrogenesis and MMP Repression}

First, we surveyed the concept of MMP repression in tissue fibrosis by a standard hepatic model. The acute liver injure was induced by a single TAA injection. As shown in Figure 1A, parenchymal hepatic damage is indicated by large scale necrosis, hemorrhage, and elevated alanine transaminase (data not shown). Liver fibrosis was induced in the mice by repeating TAA injection for 8 weeks, shown by the formation of polygonal fibrotic septa in Sirius Red staining (Figure 1A) and validated by increased mRNA level of interstitial type-I collagen (Figure 1B). Activities of MMP2 and MMP9 in the liver tissues were measured by gelatinolytic zymography (Figure 1C). In uninjured normal livers, MMP9 expression was totally absent, while MMP2 was constitutively expressed at low level (data not shown). As expected, a massive amount of MMP9 was promptly induced in the acute injured liver (the identity of MMP9 in the zymography has been previously confirmed by Western blot analysis) ${ }^{15}$. In drastic contrast, no detectable MMP9 activities were found in the fibrotic liver under injury, while a low level of MMP2 was constitutively present in both groups. Consistent with this protein expression pattern, mRNA of MMP9 was greatly induced in the acute injured group, but was barely detected in fibrotic livers (about $90 \%$ of reduction) (Figure 1D). We then expanded the study to include MMP12 (macrophage elastase), MMP13 (type-I collagenase),
A

final injection (TAA)

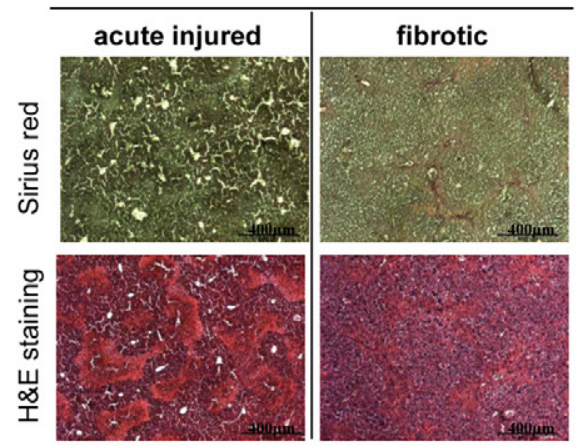

B

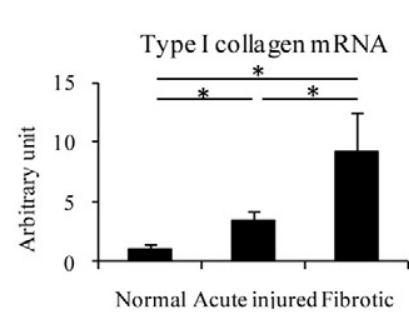

C

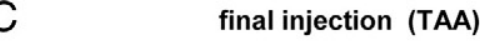

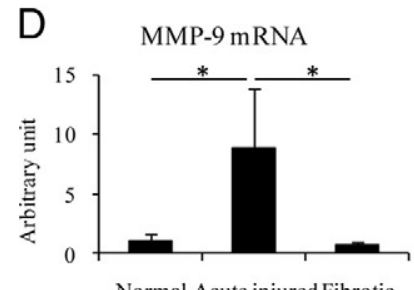

Normal Acute injured Fibrotic

MMP-13 mRNA

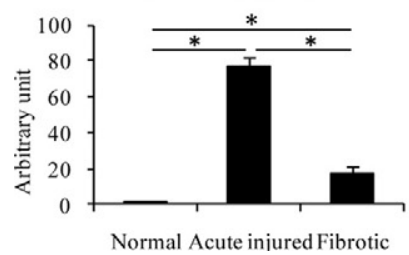

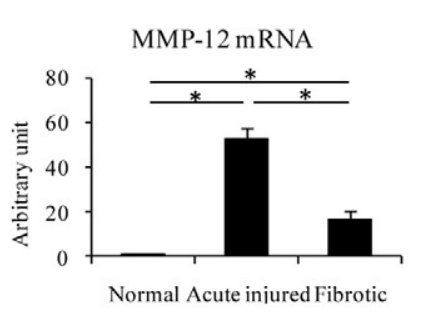

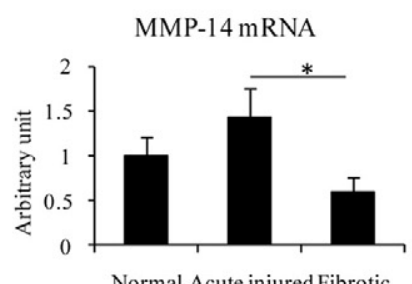

E
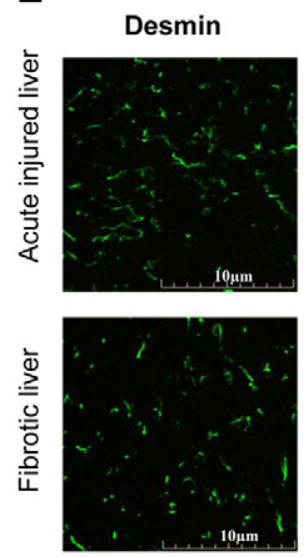

MMP-9
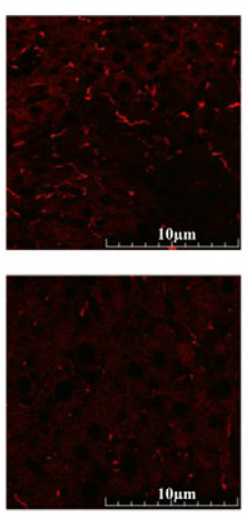

Fibrotic

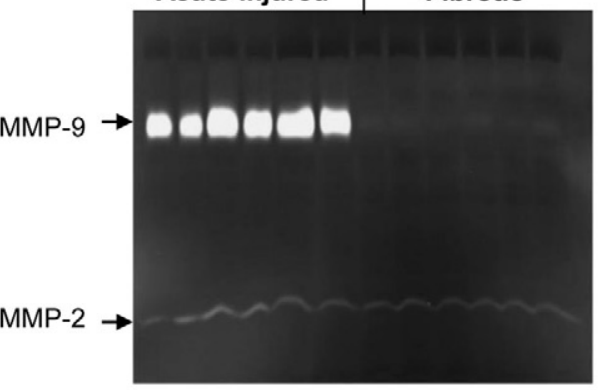

Figure 1. MMPs are induced in acute liver injury and repressed in fibrosis. Acute liver injury was induced by intraperitoneal injection of TAA at 0.2 mg/g body weight $(n=6)$. The mice were sacrificed in 24 hours. Fibrotic liver was created by repeating injection of TAA, twice a week for 8 weeks $(n=6)$. One day before sacrifice, the mice were subjected to a final TAA challenge. Normal control mice were injected with the same volume of saline. A: Liver fibrosis was measured by Sirius Red staining, and liver injury is indicated by H\&E staining. B: Liver fibrosis was also confirmed by increased expression of type-I collagen as measured by qRT-PCR. The expression levels were normalized to GAPDH mRNA levels and presented as the fold mRNA level in fibrotic livers or in acute injured livers compared to that in normal livers. The results are the mean values with standard deviations. ${ }^{*} P<0.05$. C: Gelatinases in liver tissues were accessed by zymography. D: The mRNA levels of MMPs 9, 12, 13, and 14 were measured by qRT-PCR and normalized to GAPDH and presented as the fold mRNA level in fibrotic livers or in acute injured livers compared to that in normal livers. The results are the mean values with standard deviations. ${ }^{*} P<0.05$. E: Liver sections were subjected to immunofluorescence staining for desmin (a marker for HSCs) and MMP9. 
A
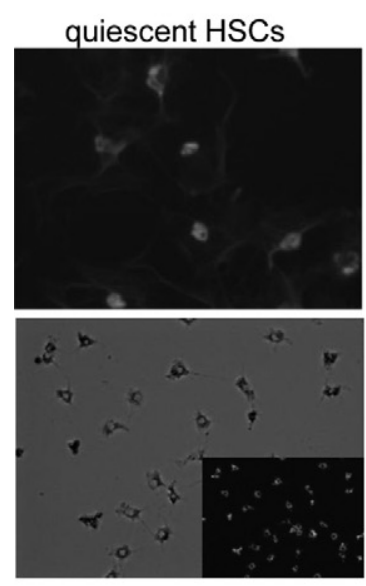

C

- quiescent HSC

$\square$ myofibroblastic HSC

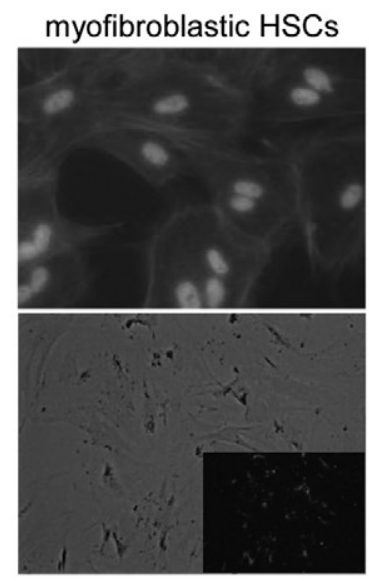

MMP-9 mRNA

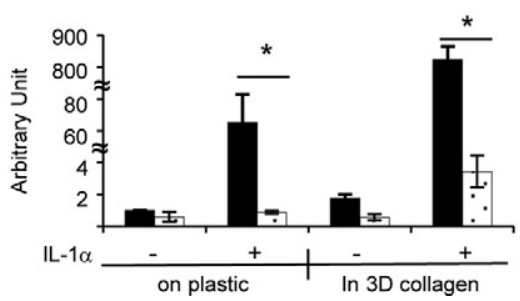

B

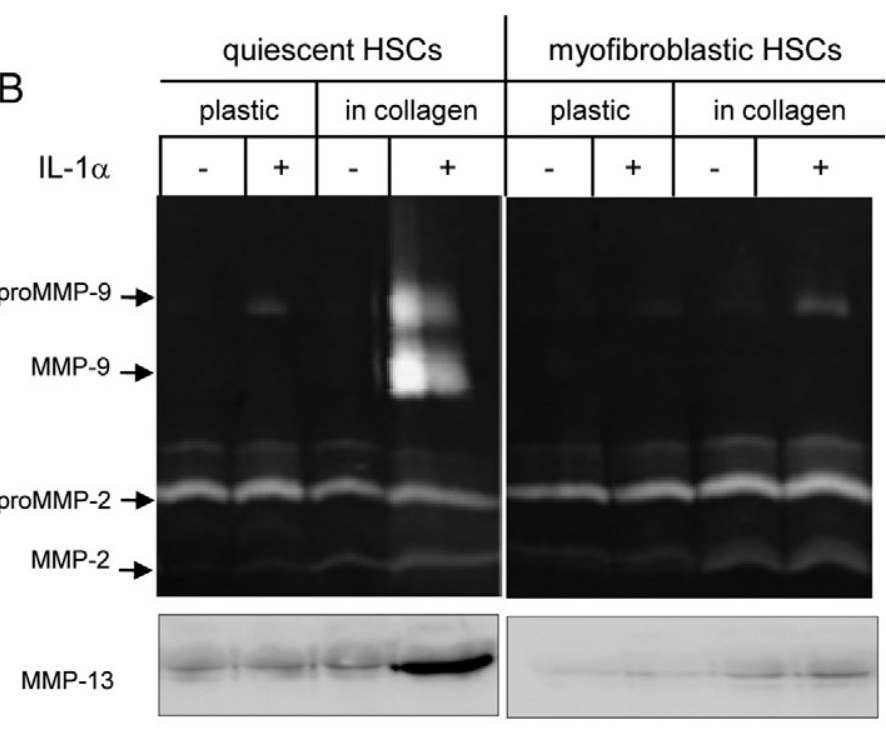

MMP-13 mRNA

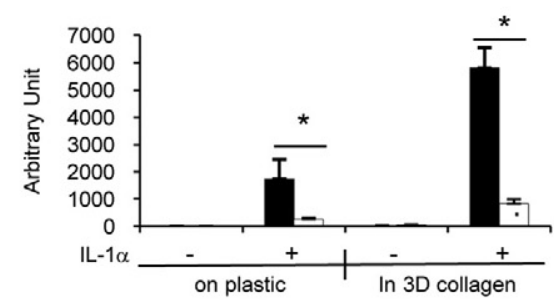

Figure 2. Repression of MMP genes during HSC transdifferentiation in vitro. Freshly isolated rat HSCs after recovery for two to three days still retain some features of a quiescent phenotype. In continuous culture on plastic up to nine to ten days, the cells transdifferentiate into myofibroblasts. A: Phenotypes of quiescent (day two) and myofibroblastic HSCs (day nine) were measured by phase-contrast microscopy and immunofluorescence staining for filamentous-actin and DNA. The insets were taken under fluorescent light to show vitamin A droplets, which are lost by myofibroblastic HSCs. B: Quiescent and myofibroblastic HSCs were seeded on plastic or embedded in 3D type-I collagen, followed by IL-1 stimulation for 3 days. Secreted MMP9 and MMP2 in the conditioned medium were revealed by gelatinolytic zymography. MMP13 was measured by Western blot analysis. C: The mRNA levels of MMP9 and MMP13 by HSCs in quiescent or myofibroblastic states were measured by qRT-PCR. Results are the average of three independent experiments, and plotted as the fold increase over basal levels. ${ }^{*} P<0.05$.

and MMP14 (membrane-type MMP). As shown in the qRT-PCR analysis, mRNA levels of these three MMPs were also significantly suppressed in the fibrotic liver (75\%, $68 \%$, and $50 \%$ of reductions respectively) compared with the acute injured liver. Massive necrosis in liver parenchyma was observed in the acute injured mice, but was absent in the fibrotic liver (Figure 1A), in a tight association with lack of hepatic MMPs, suggesting toxic roles of MMPs in tissue injury.

In the liver, HSCs are the major sources of both ECM and MMP. ${ }^{34}$ Such proximity of enzymes (MMPs) and substrates (ECM) suggests a potential role of HSCs in hepatic injury. ${ }^{8}$ We investigated the contribution of HSCs to hepatic MMP expression by double immunofluorescent staining. HSCs, as identified by desmin staining, exhibited cytoplasmic extensions, condensed cell body, and existed in a population of 8 to $12 \%$ (Figure 1E). Consistent with our prediction, MMP9 and MMP13 (not shown) were mostly colocalized with the desmin-positive cells, indicating HSCs as the major hepatic source of these two MMPs in acute liver injury. Of note is that the number of MMP-positive cells in fibrotic livers was substantially reduced, in line with the zymography data. In summary, MMP genes in normal livers are transcriptionally permissive or inducible in response to acute injury.
As a consequence of chronic injury, most MMP genes are progressively repressed or silent in fibrotic livers in favor of ECM accumulation.

\section{MMP Repression in Transdifferentiation of HSCs in Vitro}

To address the mechanism of MMP silencing in liver fibrosis, we developed an in vitro system which recapitulated the key characteristics of MMP expression in acute injured livers, and their repression in fibrotic livers. Freshly isolated rat HSCs remain quiescent for 2 to 3 days, during which the cells display ample vitamin-A droplets (visualized under UV excitation) and appear star-like phenotypes with condensed nuclei and cell bodies (Figure 2A). After cultured on plastic for 9 to 10 days, quiescent cells are morphologically and biochemically transformed to myofibroblast-like cells, as indicated by loss of vitamin-A droplets, buildup of filamentous actin fibers, gain of contractibility, and cell size expansion. To examine their potentials to express MMPs, we seeded the quiescent and myofibroblastic HSCs on plastic. And to assess the contribution of the 3D ECM to MMP expression, we also embedded the cells in type-l collagen, 
MMP-9 mRNA by quiescent HSCs on plastic

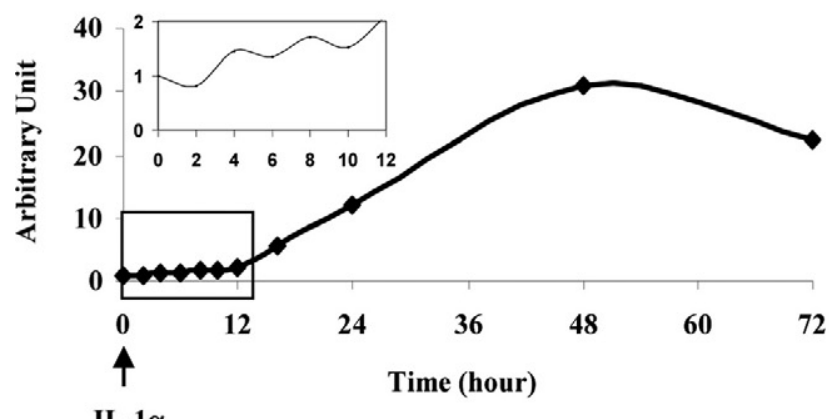

MMP-13 mRNA by quiescent HSCs on plastic

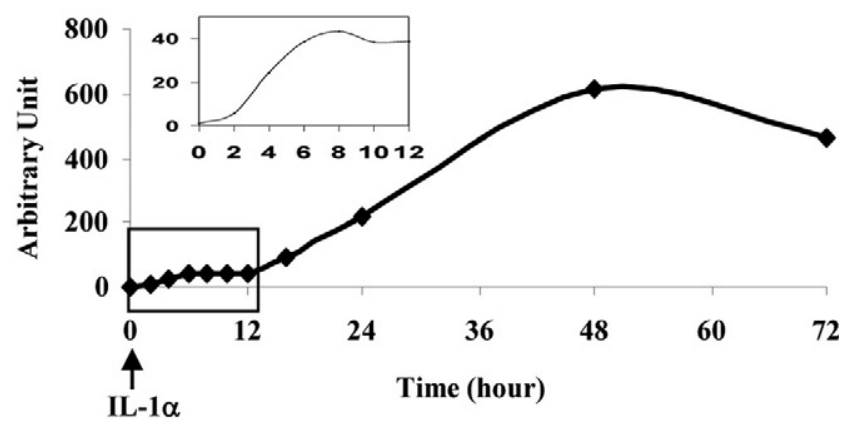

Figure 3. Dual-phase kinetics of MMP transcription in HSCs. Quiescent HSCs were stimulated by IL- 1 and harvested at the indicated time points. The mRNA levels of MMP9 and MMP13 in quiescent HSCs were measured by qRT-PCR. The results are plotted as fold induction over basal. The fine resolution of the early time course was plotted in boxes. The results are representative of three independent experiments, giving similar results.

which simulated the microenvironment in the space of Disse. The cultures were stimulated with or without IL-1 for 3 days. Choice of IL-1 as the major injury signal was previously determined by testing a panel of inflammatory cytokines and growth factors (data not shown). As shown in Figure 2B, quiescent HSCs produced abundant proMMP9 (115 kDa for rodent) in response to IL-1, and 3D collagen greatly increased proMMP9 as well as its maturation to active MMP9 (105 kDa). A similar pattern for MMP13 production by quiescent HSCs was also observed, implying that a common regulation may apply to both MMP genes. In contrast, myofibroblastic HSCs barely expressed MMP9 and MMP13 even in the 3D culture stimulated by IL-1, while the fibrotic HSCs were still capable of expressing both proMMP2 (72 kDa) and active MMP2 (62 kDa). We then assessed if the MMP repression in myofibroblastic HSCs is conferred at transcriptional levels. We focused on the MMP genes in an activation cascade which we previously proposed, in an order of MMP14>MMP13>MMP9. ${ }^{35}$ As expected, the mRNA levels of MMPs 9, 12, 13, and 14 were markedly increased after 20 hour of IL-1 stimulation (Figure 2C and data not shown). In contrast, myofibroblastic HSCs colossally lost potential to express these MMPs, even in the 3D culture challenged by IL-1. Taken together, we could manipulate the culture conditions to recapitulate the basic characteristics of quiescent HSCs to express MMPs in response to injury signals; and we also showed that the myofibroblastic HSCs are incapable of expressing MMPs in the same way as the fibrotic liver.

\section{Dual Phase Kinetics of MMP Transcription in HSCs}

Having established the in vitro model, we explored the underlying mechanism of MMP silencing during HSC transdifferentiation. To study the temporal control of MMP expression in HSCs, we measured the kinetics of MMP transcription by the quiescent HSCs in response to IL-1. The mRNA levels of MMP9 and MMP13 were induced upon IL-1 stimulation in a unique two-phase mode (Figure $3)$. In the first 12 hours, mRNA levels of MMP9 and MMP13 were steadily increased at a slow rate, to 2- and 40-fold the basal level, respectively. In the next 12 hours, the mRNA levels of MMP9 and MMP13 rose dramatically to 30- and 600-fold of their basal levels, respectively. Under the condition by which HSCs were embedded in a collagen gel and challenged by IL-1, the mRNA levels of these two MMPs were increased mostly in the first 12 hours (data not shown). In myofibroblastic HSCs, transcription of MMP9 and MMP13 was at the minimal levels even under the dual stimulation by collagen plus IL-1 (data not shown). Thus, an immediate question is what happens during the time between signal activation and efficient transcription by the HSCs in permissive (quiescent) vs. nonpermissive (myofibroblastic) states.

\section{c-Jun Is Unable to Dock onto the MMP Promoters by Myofibroblastic HSCs Despite Active Upstream Signaling}

Compelling evidence has revealed nuclear factor $(\mathrm{NF})-\kappa \mathrm{B}$ and activator protein (AP)-1 as two crucial transcription factors for MMP expressions in response to cytokine stimulation. ${ }^{36,37}$ We speculated these signaling pathways might be defective or impaired in the myofibroblastic HSCs, which may sufficiently explain the MMP repression. To this regard we first investigated the signaling pathways of JNK (phosphorylation), and IKK (as indicated by degradation of its inhibitor, $I_{\kappa} \mathrm{B} \alpha$ ). The quiescent HSCs (day 2 cells) and myofibroblastic HSCs (day 9 cells) were seeded on plastic or embedded in 3D collagen and challenged by IL-1. Under the stimulation, phosphorylation of JNK and degradation of $\mid \kappa \mathrm{B} \alpha$ occurred in 5 to 15 minutes to the similar extents by the quiescent and myofibroblastic HSCs (Supplemental Figure 1 at $h$ ttp://ajp.amjpathol.org). With previous reports on the role of ERK in MMP inductions, ${ }^{38,39}$ we also examined the activation of ERK. We noticed that ERK pathway was highly active in myofibroblastic cells in response to IL-1, whereas the MMP genes were silenced. Thus, silencing of MMPs in myofibroblastic HSCs is not due to the defects in the signal transduction pathways leading to $\mathrm{NF}-\kappa \mathrm{B}$ and $\mathrm{AP}-1$ activation.

Among the four kinase pathways (JNK, IKK, p38MAPK, and $E R K$ ) initiated by IL-1, JNK is the major signal re- 
Rat MMP-9 proximal promoter

A

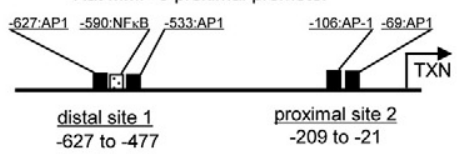

B

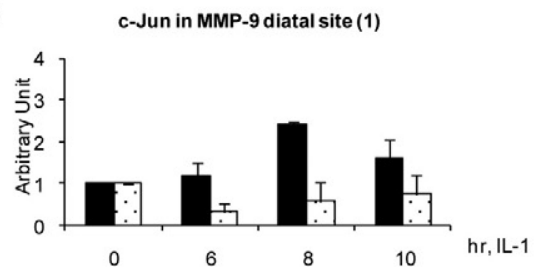

C

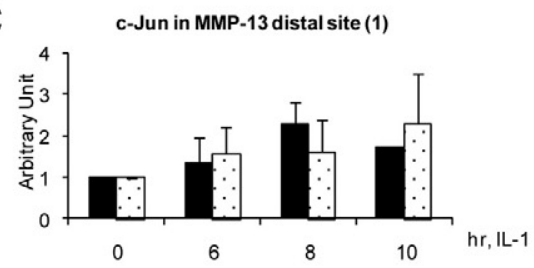

Rat MMP-13 proximal promoter

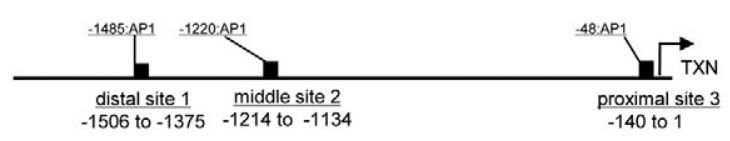

C-Jun in MMP-9 proximal site (2)

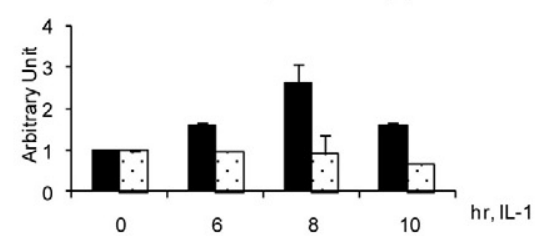

C-Jun in MMP-13 middle site (2)

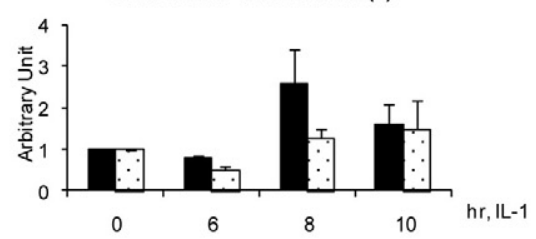

C-Jun in MMP-13 proximal site (3)

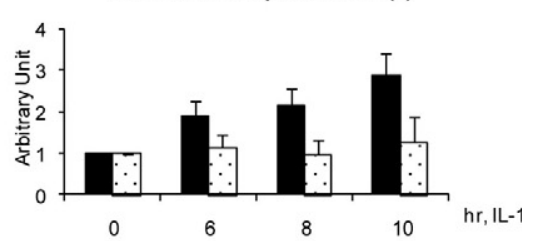

D

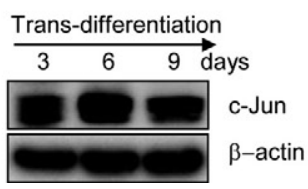

Figure 4. Time courses of recruitment of c-Jun to rat MMP9 and MMP13 promoters in quiescent and myofibroblastic HSCs. A: Schematics of rat MMP9 and MMP13 gene promoters are illustrated. Indicated are potential cis-elements for NF- $\kappa \mathrm{B}$ and AP-1 and the locations of the primer sets used for ChIP assay. Binding of c-Jun to the $5^{\prime}$-promoters of MMP9 (B) and MMP13 (C) genes by quiescent versus myofibroblastic HSCs was measured by ChIP assay followed by quantitative PCR analysis. Data were calculated as output/input ratios and further converted to fold changes over basal levels. Results are the average of three experiments with standard deviations. D: Expression of c-Jun during transdifferentiation was measured by Western blot.

quired for activation of many MMP genes. ${ }^{40}$ Using ChIP assay, we measured the recruitment of C-Jun, a prominent member of AP-1 family, onto the proximal promoters of MMP9 and MMP13 genes in the quiescent and myofibroblastic HSCs in response to IL-1 stimulation. The $5^{\prime}-$ promoter of rat MMP-9 gene has three AP-1 sites at -533 (TGAGTCA), -106 (TGAGTCA), and -69 (TGAtTCA), grouping into two loci, referring as the distal and proximal cis-elements (Figure 4A). As measured in a time course experiment, it took approximately 6 hours to recruit c-Jun onto the proximal cis-elements in MMP9 (site 2) and MMP13 (site 3) genes in the IL-1-stimulated quiescent HSCs (supplemental Figure 2 at http://ajp.amjpathol.org). Robust logging of c-Jun onto MMP9 promoter was found around 8 hours after stimulation. Such slow pace of recruitment of c-Jun to its promoter is consistent with the two-phase kinetics of MMP9 gene transcription (Figure 3). In myofibroblastic HSCs, C-Jun was barely recruited to the cis-elements in MMP9 promoter after IL-1 stimulation (Figure 4B), suggesting the MMP9 gene is physically inaccessible in the myofibroblastic cells.

Similarly, the promoter of rat MMP-13 gene has three AP-1 sites, among which two match perfectly to the consensus sequence, located at -1485 (TGAGTCA) and -48 (TGACTCA), while the third one with a highly conserved sequence resides at -1220 (TGACaCA) (Figure 4A). In the quiescent HSCs and under IL-1 stimulation, initial recruitment of c-Jun to those cis-elements was in a slow pace, in line with the slow of transcription in the first 12 hours (Figure 4C and Figure 3). In the distal region, c-Jun could bind to the cis-element in the MMP13 gene to a similar extent in both the quiescent and myofibroblastic
HSCs under IL-1 stimulation (Figure 4C). However, in the proximal region to transcription start site of the MMP13 promoter, c-jun binding was totally absent in the myofibroblasts after the IL-1 stimulation. Using Western blot analysis, we found that c-Jun protein was equally expressed in the quiescent and myofibroblastic HSCs (Figure 4D). Such results excluded a possibility that the inefficient recruitment of c-Jun might be due to it compromised expression during HSC transdifferentiation. We also measured the recruitment of p65 NF- $\kappa \mathrm{B}$ to the MMP promoters of HSCs in quiescent and myofibroblastic states. In quiescent HSCs, the binding of p65 to the MMP promoters was apparently in oscillation, in line with previous report, ${ }^{41}$ while this binding in the myofibroblasts was impaired (data not shown). Taken together, these results demonstrate that although the essential signals are intact in the myofibroblastic HSCs, the MMP promoters are inaccessible to the transcription factors. Such inaccessibility in MMP genes underscores a possible epigenetic repression in the MMP genes at chromatin levels, which is presumably built up during the transdifferentiation.

\section{Histone Acetylation and Assembly of Transcription Machinery on the MMP Promoters Are Impaired in Myofibroblastic HSCs}

To examine the hypothesis of epigenetic repression of MMPs during HSC transdifferentiation, we then investigated the level of histone acetylation and assembly of transcription machinery on MMP9 and MMP13 promoters 
A
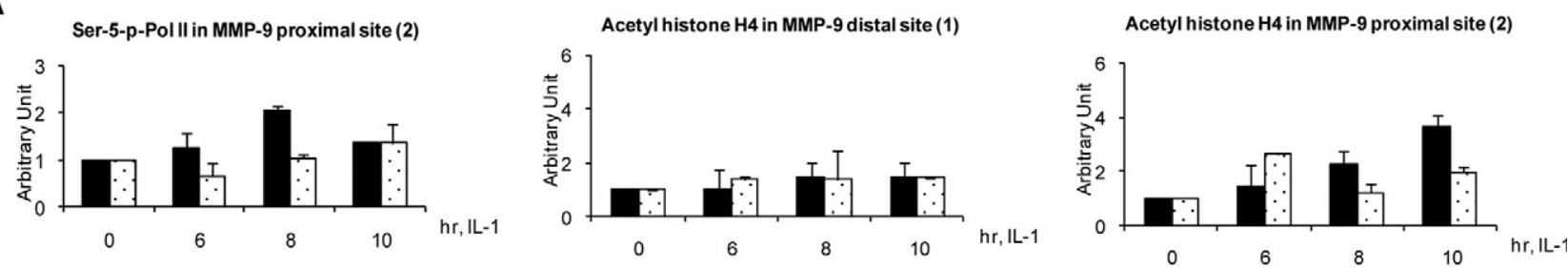

B
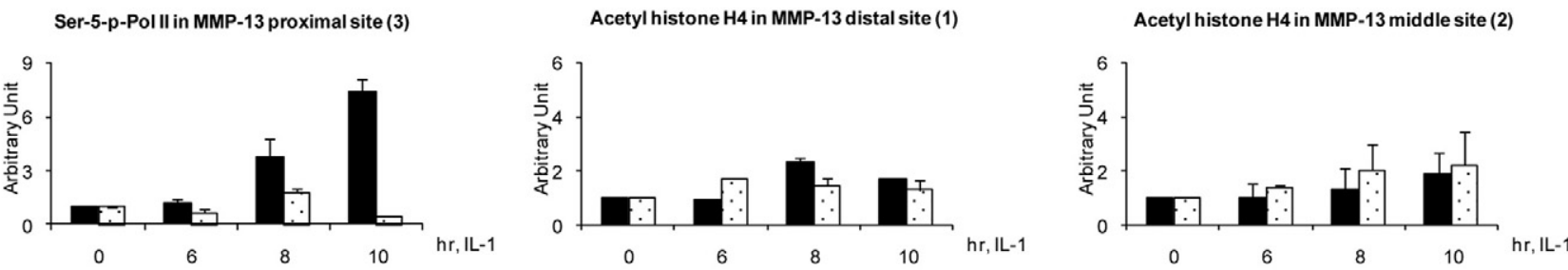

Acetyl histone $\mathrm{H} 4$ in MMP-13 proximal site (3)

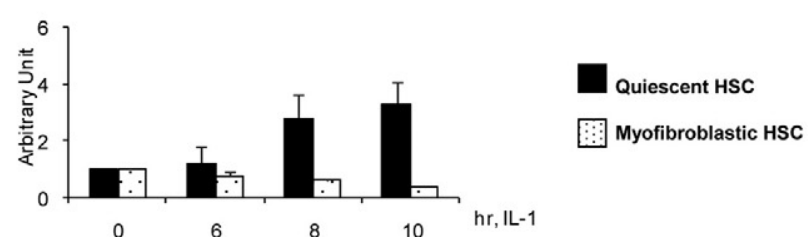

Figure 5. Impaired histone acetylation and failed recruitment of RNA Pol II to the 5'-promoters of MMP genes in myofibroblastic HSCs. Quiescent and myofibroblastic HSCs were stimulated by IL-1 and harvested at the time points indicated. Binding of Ser-5-p-Pol II and acetylation of histone H4 in MMP9 (A) and MMP 13 (B) promoters were measured by ChIP assay followed by qPCR analysis. Data were calculated as output/input ratios and further converted to fold changes over basal levels. Results are averages of three experiments with standard deviations.

(located in distal arms of chromosome \#3 and \#8 respectively). In the quiescent HSCs, N-terminal acetylation of histone $\mathrm{H} 4$ in MMP9 and MMP13 promoters was stimulated by $\mathrm{IL}-1$, and mostly occurred around the TSS (Figure 5, A and B), in accordance with the levels of $\mathrm{C}$-Jun binding at the same site. The timing of stimulated histone acetylation also coincided with the C-Jun occupancy on the MMP gene promoters after IL-1 stimulation, indicating the burst of acetylation in the region around TSS is mediated by docking of c-Jun (Figures 4 and 5). Conversely, in the myofibroblasts, docking of c-Jun was largely impaired, also in line with the failed acetylation of histone in the MMP promoters. The largest subunit of RNA Pol II becomes highly phosphorylated in transcription, and phosphorylation at Ser-5 in the repetitive carboxy-terminal domain is believed in association with start of transcription. ${ }^{42}$ We found that after stimulation by IL-1 for 6 to 8 hours, Ser-5 phosphorylated RNA Pol II was steadily recruited to the transcription start site of the two MMP promoters in the quiescent HSCs. However, such recruitment was absent in the myofibroblasts. Taken together, these results showed that the two MMP genes in myofibroblastic HSCs are transcriptional nonpermissive, due to inaccessibility to transcription factors and impaired histone acetylation, ultimately leading to insufficient loading of RNA Pol II for transcription.

\section{Histone Acetylation Is Reduced during HSC Transdifferentiation}

Switch of transcriptionally active genes into silent ones during trans-differentiation is often mediated by epigenetic switch. ${ }^{29}$ As a general rule, elevated acetylation in

the $\mathrm{N}$-terminus of histone $\mathrm{H} 3$ and $\mathrm{H} 4$ is related to active gene transcription, while impaired histone acetylation is consistently associated with permanently silenced genes in compacting heterochromatin, and in the early phase of epigenetic repression of regulated genes. To this end, we tried to address how the defective histone acetylation occurs in MMP promoters in the myofibroblastic HSCs. First, we examined histone acetylation during transdifferentiation. As shown, $\alpha$-SMA was markedly increased during transdifferentiation (Figure 6, A and B). In line with the transdifferentiation, the global levels of $\mathrm{N}$-terminal acetylation of both histone $\mathrm{H} 3$ and $\mathrm{H} 4$ were progressively reduced. We further revealed that the levels of acetyl histone $\mathrm{H} 4$ at lysine 8 (acH4K8) and 12 (acH4K12) were down-regulated during the HSC trans-differentiation, while acetylation at lysine 16 of $\mathrm{H} 4$ (acH4K16) was slightly increased, and acetylation at lysine 5 (acH4K5) was barely detected even in the quiescent HSCs. To further confirm these results, we fractionated HSCs into cytoplasmic and membrane/nucleus (containing nuclear components) pools, characterized by GAPDH and lamin $\mathrm{A} / \mathrm{C}$, the cytoplasmic and nuclear marks, respectively (Figure 6C). In membrane/nuclear fractions, acetylated $\mathrm{H} 3$ and $\mathrm{H} 4$ clearly decreased in the course of transdifferentiation. Histone methylation (H3K9me3) at the global level was not significantly changed during the early transdifferentiation, and was slightly reduced at the final stage. Finally, using quantitative ChIP assays, we measured the basal acetylation around the TSS in MMP13. As shown in Figure 6D, the basal level of histone acetylation in MMP13 was significantly impaired in the myofibroblastic HSCs, further demonstrating the condensed nucleosome in MMP genes in the myofibroblastic HSCs. Therefore, impaired histone acetylation 
A

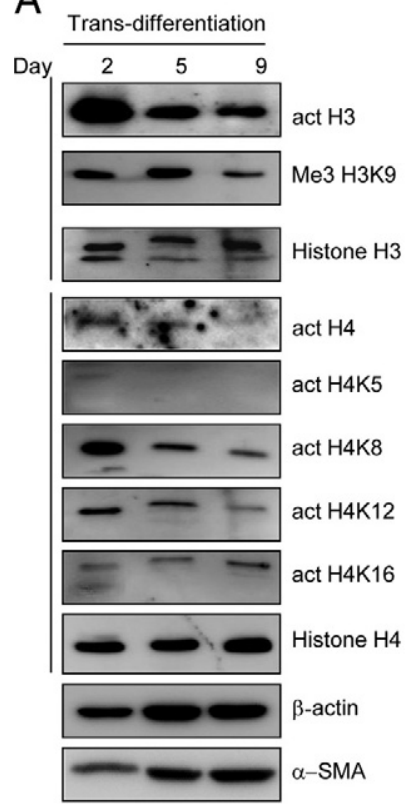

B
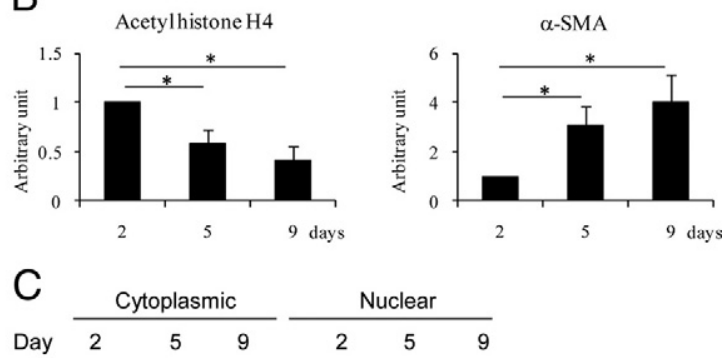

Day

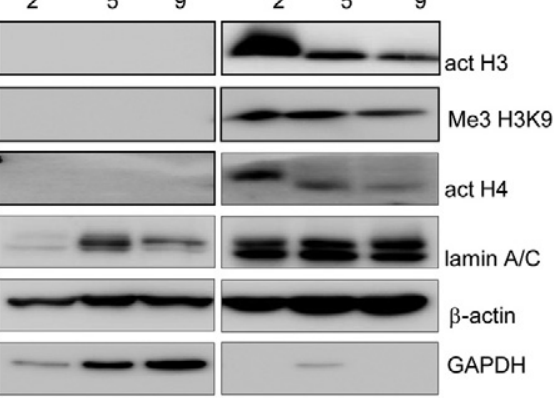

D Acetyl Histone H4 MMP13 proximal site (3)

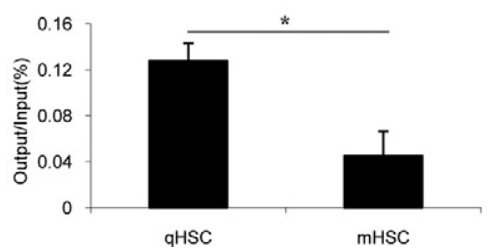

Figure 6. Reduced histone acetylation during HSC transdifferentiation. A: Quiescent (day two), intermediate (day five), and myofibroblastic (day nine) HSCs were examined for $\mathrm{H} 3$ and $\mathrm{H} 4$ histone acetylation and methylation by Western blot analysis. Transdifferentiation was monitored by increased expression of $\alpha$-SMA. B: Quantification of acetyl histone $\mathrm{H} 4$ and $\alpha$-SMA by Western blot was measured by densitometry, representing three independent experiments. $\mathbf{C}$ Cells were fractionated into cytoplasmic and nuclear/membrane fractions. Decreased acetylation of histone $\mathrm{H} 3$ and $\mathrm{H} 4$ during transdifferentiation is obvious. D: In quiescent (day two, qHSC) and myofibroblastic (day nine, mHSC) HSCs, basal levels of acetyl histone H4 in the transcription start site of MMP13 gene were measured by ChIP assay and qRT-PCR. Data represent the output/input ratios and are the averages of three independent experiments. Statistical significance, ${ }^{*} P<0.05$. occurred at the MMP promoters and globally during transdifferentiation.

\section{HDAC4 Accumulates during Transdifferentiation}

The dynamic level of histone acetylation in cells is maintained by opposite actions of HATs and HDACs. During transcription, HAT bearing co-activators are recruited to promoters, mediated through transcription factors, such as $\mathrm{AP}-1$ and NF- $\kappa \mathrm{B}$. Since the signaling pathways for AP-1 and NF- $\kappa$ B activation were intact in the myofibroblastic HSCs, we reasoned that the reduction in the level of histone acetylation in the myofibroblastic HSCs would be more likely through elevated histone deacetylation. To this regard, we surveyed the expression of class I and II HDACs during HSC transdifferentiation. Protein levels of class I HDACs such as HDAC1, 2, and 3 were not significantly changed (Figure 7A). In contrast, HDAC4, members of class II HDAC, was steadily increased (Figure 7B), while HDAC5 and 7, another two class II HDACs, were barely detected by the Western blotting. By immunofluorescent staining, we found HDAC4, which was distributed in both the nucleus and cytoplasm, was significantly increased during myofibroblastic activation (Figure 7C). Finally, we examined induction of HDAC4 during HSC activation by an animal model. On injection of carbon tetrachloride, HSCs were initially activated as marked by expression of $\alpha$-SMA and synthesis of interstitial collagen (data not shown). HSCs, indicated as desmin positive cells, barely expressed HDAC4 in the normal liver (Figure 7D). As a consequence of initial cellular activation the HDAC4 was clearly increased and colocalized in the HSCs, which provides a pathophysiological relevance. Taken together, these results suggest that HDAC4 accumulation may associate with repression of MMP genes in liver fibrosis.

\section{Ectopic Expression of HDAC4 in Quiescent HSCs Suppresses the Promoter Activities of MMPS}

To demonstrate causal relationship of HDAC4 elevation in MMP gene repression, we ectopically expressed HDAC4 in the quiescent/permissive HSCs and examined its effects on MMP gene expression. Primary HSCs, 2 days after isolation, were cotransfected with MMP expression reporter plasmids (1.3-kb 5'-MMP9 promoter and 1.8-kb 5'-MMP13 promoter, respectively), together with an expression plasmid for the mouse HDAC4 gene. After transfection, cells were stimulated with IL-1 for additional 18 hours. As shown in Figure 8A, IL-1 significantly stimulated promoter activities of both MMP9 (1.8-fold) and MMP13 (2.9-fold). Ectopic expression of HDAC4 completely abolished the IL-1-mediated induction of the promoter activities. In addition, endogenous MMP9 expression, at the protein level, was partially repressed by HDAC4 overexpression in the transient transfection (Figure 8, B and C). As control, both proMMP2 and its mature form were not affected by HDAC4 ectopic expression. Expression 
A

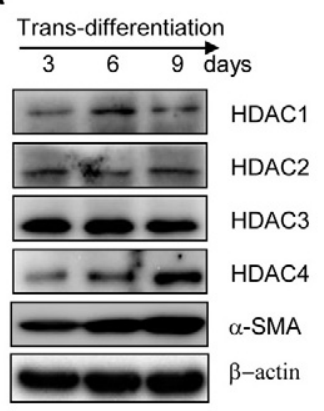

B
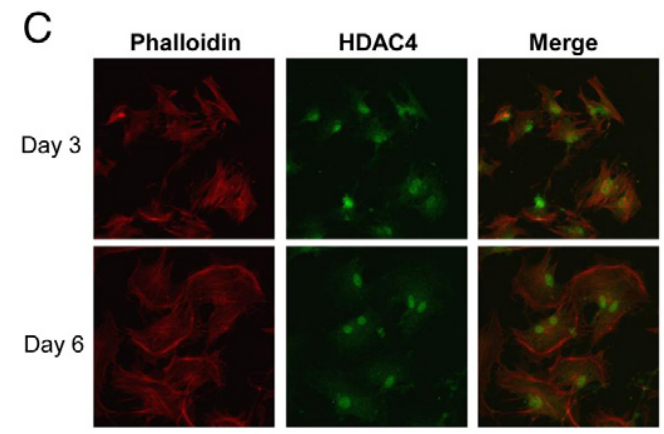

Day 9
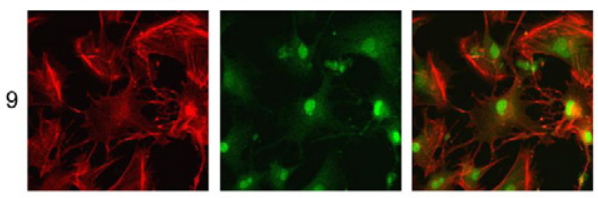

D control rat liver rat liver 4 days after $\mathrm{CCl}_{4}$ injection

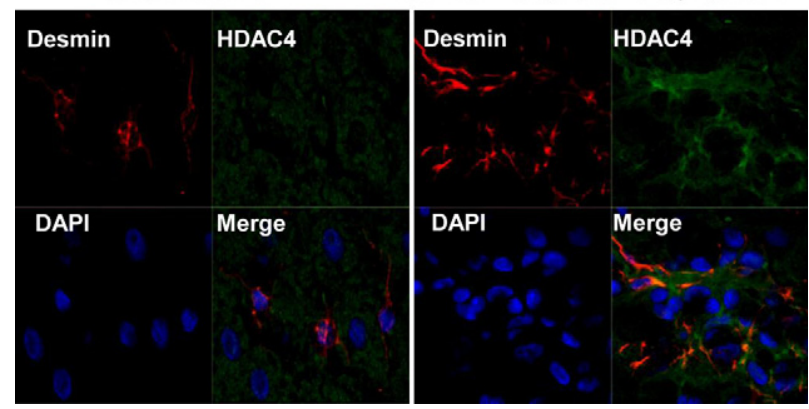

Figure 7. Accumulation of HDAC4 during HSC transdifferentiation. A: Expression levels of class I (HDAC1, $-2,-3$ ) and II (HDAC4) by HSCs during transdifferentiation were measured by Western blot analysis. Transdifferentiation of HSCs was monitored by increased expression of $\alpha$-SMA. B: Quantification of HDAC4 was measured by densitometry scanning of Western blot, representing three independent experiments. Statistical significance, ${ }^{*} P<$ 0.05 . C: Distribution of HDAC4 in nuclear and cytoplasmic compartments during HSC activation is shown by immunofluorescence staining. D: Expression of HDAC4 (green) in HSCs [marked by desmin (red)] of rat liver was examined by immunofluorescence staining.

of exogenous HDAC4 in the stellate cells was confirmed by Western blot analysis (Figure 8D).

\section{Discussion}

In actively growing tissues such as bone marrows and tumors, as well as injured tissues, many MMPs are highly expressed, presumably to facilitate growth factor release, cell activation, proliferation, migration, and differentiation. ${ }^{43,44}$ Uncontrolled surges of MMPs in fulminate hep- atitis are toxic and even lethal, while persistent elevation of MMPs is a key feature of chronic wounds and other degenerative diseases such as arthritis and tumor metastasis. ${ }^{8,45}$ The pathophysiological expression of MMPs in tissues is subject to tight control at various levels, from the extracellular stimuli including multiple cytokines and the surrounding ECM to the status quo of the cells in their epigenetic stages. Initially observed by Friedman et al ${ }^{46}$ and Arthur et $\mathrm{al}^{47}$ in Bissell's lab, HSCs are fascinating for their dual roles to secret both ECM and their degradation enzymes, MMPs. However, apparent dilemma is that how HSC can avoid producing MMPs and ECM at the same time.

In this study we proposed a novel model to explain how MMP genes are epigenetically repressed during HSC transdifferentiation in liver fibrosis. As depicted in Figure 9, the quiescent phenotypes of HSCs in the normal liver are maintained by the loose 3D ECM produced by the stellate cells themselves. Intracellular vitamin A storage and extracellular basement membrane ECM are the two major characteristics of the quiescent phenotypes in the normal liver. The MMP genes in the quiescent HSCs are transcriptionally permissive or inducible, in part, related to the presumably accessible chromatin configurations at the MMP loci. On stimulation of injury signals such as IL-1, stress kinases JNK and IKK are activated, which results in recruitment of transcription co-activators bearing HAT activities. Histone acetylation in the proximal region to TSS may facilitate the binding of RNA polymerase II to the MMP promoters. Such permissive state in quiescent HSCs and accessibility in MMP genes are associated to elevation of histone acetylation at both global and gene specific levels. As the consequence of transdifferentiation, the MMP genes in HSCs are epigenetically silenced to favor ECM accumulation in fibrosis.

As shown in Figure 1A, the fibrotic liver is resistant to chemical injury, implying impaired inflammation, which may consequently result in lacking of MMP expression. For this regard, we measured the mRNA levels of inflammatory cytokines, including IL-1, TNF- $\alpha$, and IL-6. Indeed, those cytokines were greatly induced in acute liver injury, and then decreased in fibrotic liver (supplemental Figure 3 at http://ajp.amjpathol.org). Therefore, it is possible that this reduction of inflammation may contribute, in part, to MMP repression in fibrotic liver. Thus, the repression of MMPs in fibrotic tissues is mediated by loss of extracellular cues and intracellular epigenetic switch.

How permissive genes are epigenetically silenced during cell differentiation is not well defined. Generally, it is believed that methylation in CpG sites or "islands" in gene promoters is related to condensed inactive chromatin. However, DNA methylation at particular loci may not be necessarily required for silencing of inducible genes in cell differentiation. For example, silencing of a transgene in a cell line was found preceded DNA and histone methylation. ${ }^{48}$ For this regard, we surveyed DNA methylation changes in the MMP9 and MMP13 genes during HSC transdifferentiation in vitro. We found the cytosine methylation in $\mathrm{CpG}$ sites in these two genes does not significantly altered during transdifferentiation (Qin et al, unpublished data). However, this does not exclude a 
A

Rat MMP-9 promoter activities

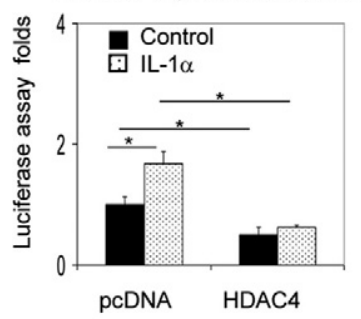

C

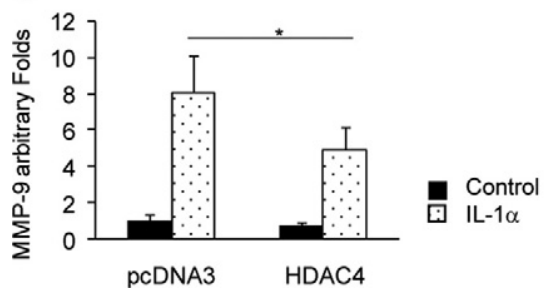

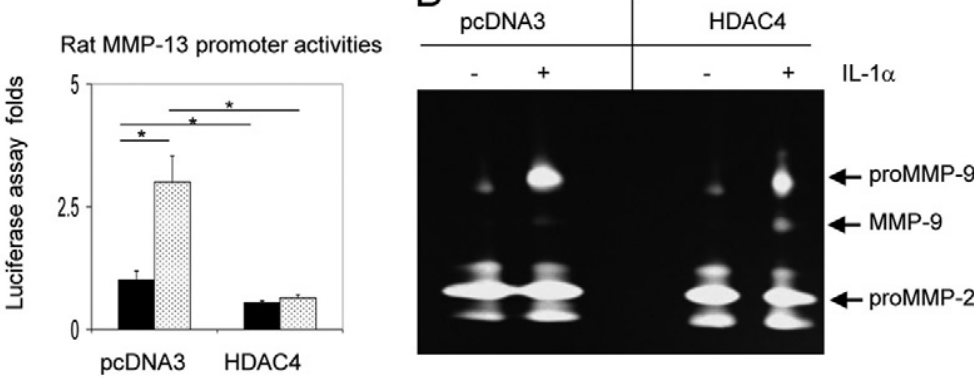

B

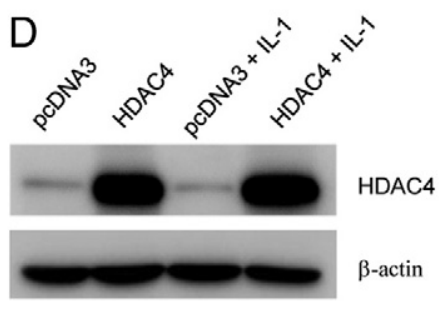

Figure 8. Ectopic expression of HDAC4 in quiescent HSCs suppresses MMP transcription. A: Quiescent HSCs were co-transfected with three plasmids: (1) reporter plasmids (p1300MMP9-luc or p1800MMP13-luc) encoding firefly luciferase driven by rat MMP9 or -13 5'-promoters, (2) an expression plasmid encoding mouse HDAC4 driven by the CMV promoter, and (3) renilla luciferase to monitor transfection efficiency. After transfection, cells were stimulated with or without IL-1 for an additional 20 hours. Dual luciferase assay was used to report MMP promoter activities. The results are averages of three replicates with standard deviations. Statistic significance was calculated by Student's $t$-test, with ${ }^{*} P<0.05$. B: Effects of ectopic expression of HDAC4 on endogenous MMP9 in quiescent HSCs were determined by transient transfection of the expression plasmid followed by IL-1 stimulation for 24 hours. Gelatinases in the conditioned medium were concentrated by gelatin-conjugated Sepharose 4B and revealed by zymography. C: Semiquantitative measurements of endogenous MMP9 protein were measured by densitometric scanning of the zymography of three replicates. D: The ectopic expression of mouse HDAC4 in HSCs was confirmed by Western blot analysis. Statistical significance, ${ }^{*} P<0.05$.

possible role of DNA methylation in the later phase repression of MMP loci, through which heterochromatin like regions may be built up in the fibrosis. Instead, our findings reported here indicate that the epigenetic repression of MMP genes may largely be regulated at the level of histone acetylation.

The N-terminal acetylation in both $\mathrm{H} 3$ and $\mathrm{H} 4$ is globally reduced in the myofibroblastic HSCs. These results promoted us to investigate the key enzymes involved in histone acetylation. Histone acetylation is a consequence of the net balance of both HATs and HDACs. HATs are brought into promoters through the incorporation of transcription factors. Initially, we speculated the inactivation of MMP genes may be simply due to dampened signaling events and/or insufficient HAT recruitment in the myofibroblastic HSCs. Compared with the quiescent HSCs, the myofibroblastic cells equally exhibited activation of the JNK/C-Jun and IKK/NF- $\kappa$ B pathways. Although being activated, the key transcription factors still failed to reach the MMP promoters, suggesting epigenetic modification occurs in the MMP genes during the transdifferentiation. Indeed, both the levels of basal and induced histone acetylation in the MMP promoters are significantly impaired in the myofibroblastic cells.

HDACs as co-repressors have been linked to epigenetic silencing. ${ }^{28}$ Although class I HDACs has been extensively studied in gene repression, we found that HDAC 1, 2, and 3 are not significantly altered during HSC transdifferentiation. Instead, we found that the expression level of HDAC4, a class II HDAC member, profoundly increase during HSC transdifferentiation. Up-regulation of HDAC4 correlates well with the reduced level of histone acetylation. In accordance, ectopic expression of HDAC4 in the HSCs at permissive state can represses the MMP genes. One question is how HDACs are recruited to chromatin in the myofibroblasts, whether in a locus specific or nonspecific manner. HDACs in general are unable to bind DNA directly; instead they connect DNA through interactions with other DNA binding proteins such as NuRD, Sin3, and CoREST. ${ }^{49,50}$ Lodging of

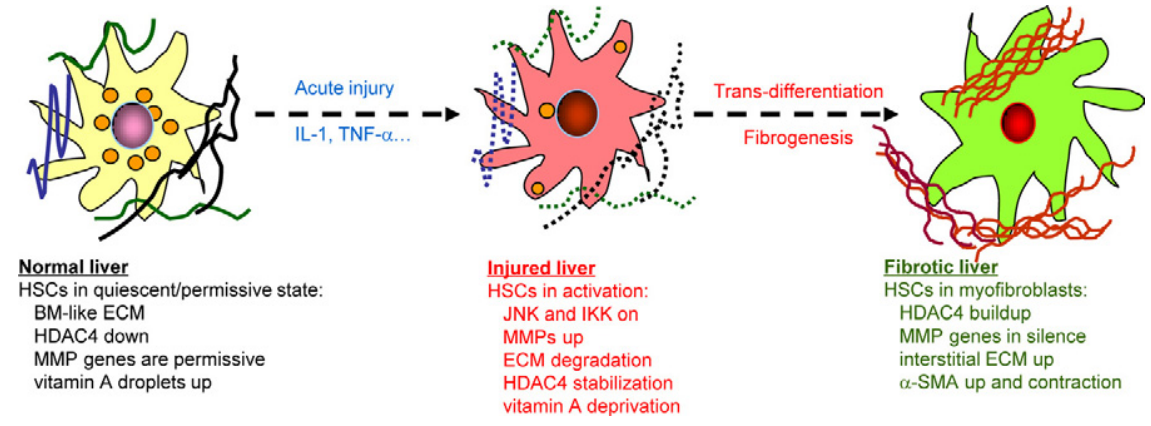

Figure 9. A model to explain how MMP genes are epigenetically permissive in quiescent HSCs and repressed during HSC transdifferentiation and liver fibrogenesis. 
HDACs in the chromatin was proposed through methyl$\mathrm{CpG}$ and its binding protein MeCP2 (methylcytosinebinding protein 2). ${ }^{51-53}$ Therefore, the timing of MMP gene silencing, histone acetylation/methylation and DNA methylation should be studied specifically in our system both in vivo and in vitro. Further, HDACs may also be recruited to DNA through their associated transcription factors and co-repressors. ${ }^{28}$ JDP2 (Jun dimerization protein 2), a repressor of AP-1 was found to recruit HDAC3 in transcription suppression. ${ }^{54}$ In addition to histones, many transcription factors are also targets of acetylation. ${ }^{55,56}$ For instance, acetylation of RelA/p65 abolishes its interaction with $I_{\kappa} \mathrm{B}$ and thus enhances its binding to DNA. ${ }^{57}$ By these accounts, elevated HDAC4 in myofibroblasts may also impair AP-1 and NF- $\kappa$ B function through deacetylation or by incorporation into the suppression complex. Our findings reported here will facilitate the further detailed investigation on how HDAC4 is induced and recruited to the MMP promoters in tissue fibrosis.

In addition to HSCs, Kupffer cells, the hepatic macrophages were also identified in liver injury. ${ }^{58}$ But the HSCproduced MMPs are special because of their proximity to the ECM within the space of Disse. Although the injured type of MMPs (including MMP9 and MMP13) is silenced in fibrotic liver, MMP2 is elevated, which may facilitate fibrosis resolution. ${ }^{59}$ On the other hand, accumulation of TIMPs may additionally account the EMC accumulation in fibrotic tissues. ${ }^{60}$

\section{Acknowledgments}

We thank Dr. Hidekazu Tsukamoto and Jiao-Hong Wang in the Southern California Research Center for Alcoholic Liver and Pancreatic Diseases and Cirrhosis for advice and providing hepatic stellate cells. We also thank Dr. Neil Kaplowitz and the USC Research Center for Liver Diseases for providing ABI 7900 DNA sequencing system and confocal microscope in the microscopy subcore. We appreciate Dr. Xinhua Feng, Dr. Mingshu Huang, Dr. Hai-Tao Yang, Daniel Purcell, and Chao Quan for their critical reading of the manuscript.

\section{References}

1. Werb Z, Chin JR: Extracellular matrix remodeling during morphogenesis. Ann NY Acad Sci 1998, 857:110-118

2. Friedman SL: Hepatic stellate cells: protean, multifunctional, and enigmatic cells of the liver. Physiol Rev 2008, 88:125-172

3. Alcolado R, Arthur MJ, Iredale JP: Pathogenesis of liver fibrosis. Clin Sci (Lond) 1997, 92:103-112

4. Bataller R, Brenner DA: Liver fibrosis. J Clin Invest 2005, 115:209-218

5. Reeves HL, Friedman SL: Activation of hepatic stellate cells-a key issue in liver fibrosis. Front Biosci 2002, 7:d808-d826

6. Geerts A: History, heterogeneity, developmental biology, and functions of quiescent hepatic stellate cells. Semin Liver Dis 2001, 21:311-335

7. Schuppan D, Ruehl M, Somasundaram R, Hahn EG: Matrix as a modulator of hepatic fibrogenesis. Semin Liver Dis 2001, 21:351-372

8. Yan C, Zhou L, Han YP: Contribution of hepatic stellate cells and matrix metalloproteinase 9 in acute liver failure. Liver Int 2008, 27:959-971

9. Yu Q, Stamenkovic I: Cell surface-localized matrix metalloprotein- ase-9 proteolytically activates TGF-beta and promotes tumor invasion and angiogenesis. Genes Dev 2000, 14:163-176

10. Reynaert H, Thompson MG, Thomas T, Geerts A: Hepatic stellate cells: role in microcirculation and pathophysiology of portal hypertension. Gut 2002, 50:571-581

11. Wells RG: The role of matrix stiffness in hepatic stellate cell activation and liver fibrosis. J Clin Gastroenterol 2005, 39:S158-S161

12. Kisseleva T, Brenner DA: Role of hepatic stellate cells in fibrogenesis and the reversal of fibrosis. J Gastroenterol Hepatol 2007, 22 Suppl 1:S73-S78

13. Rockey DC, Weisiger RA: Endothelin induced contractility of stellate cells from normal and cirrhotic rat liver: implications for regulation of portal pressure and resistance. Hepatology 1996, 24:233-240

14. Parsons CJ, Takashima M, Rippe RA: Molecular mechanisms of hepatic fibrogenesis. J Gastroenterol Hepatol 2007, 22 Suppl 1:S79-S84

15. Han YP, Zhou L, Wang J, Xiong S, Garner WL, French SW, Tsukamoto $\mathrm{H}$ : Essential role of matrix metalloproteinases in interleukin-1-induced myofibroblastic activation of hepatic stellate cell in collagen. J Biol Chem 2004, 279:4820-4828

16. Han YP, Tuan TL, Hughes M, Wu H, Garner WL: Transforming growth factor-beta - and tumor necrosis factor-alpha - mediated induction and proteolytic activation of MMP-9 in human skin. J Biol Chem 2001, 276:22341-22350

17. Zhou L, Yan C, Gieling RG, Kida Y, Garner W, Li W, Han YP: Tumor necrosis factor-alpha induced expression of matrix metalloproteinase-9 through p21-activated kinase-1. BMC Immunol 2009, 10:15

18. Wielockx B, Lannoy K, Shapiro SD, Itoh T, Itohara S, Vandekerckhove J, Libert C: Inhibition of matrix metalloproteinases blocks lethal hepatitis and apoptosis induced by tumor necrosis factor and allows safe antitumor therapy. Nat Med 2001, 7:1202-1208

19. Gum R, Lengyel E, Juarez J, Chen JH, Sato H, Seiki M, Boyd D: Stimulation of $92-\mathrm{kDa}$ gelatinase $\mathrm{B}$ promoter activity by ras is mitogen-activated protein kinase kinase 1 -independent and requires multiple transcription factor binding sites including closely spaced PEA3/ ets and AP-1 sequences. J Biol Chem 1996, 271:10672-10680

20. Eberhardt W, Akool el S, Rebhan J, Frank S, Beck KF, Franzen R, Hamada FM, Pfeilschifter J: Inhibition of cytokine-induced matrix metalloproteinase 9 expression by peroxisome proliferator-activated receptor alpha agonists is indirect and due to a NO-mediated reduction of mRNA stability. J Biol Chem 2002, 277:33518-33528

21. Verdone L, Caserta M, Di Mauro E: Role of histone acetylation in the control of gene expression. Biochem Cell Biol 2005, 83:344-353

22. Clayton AL, Hazzalin CA, Mahadevan LC: Enhanced histone acetylation and transcription: a dynamic perspective. Mol Cell 2006, 23:289-296

23. O'Neill LP, Turner BM: Histone $\mathrm{H} 4$ acetylation distinguishes coding regions of the human genome from heterochromatin in a differentiation-dependent but transcription-independent manner. EMBO J 1995, 14:3946-3957

24. Ausio J, van Holde KE: Histone hyperacetylation: its effects on nucleosome conformation and stability. Biochemistry 1986, 25:1421-1428

25. Vermeulen M, Walter W, Le Guezennec X, Kim J, Edayathumangalam RS, Lasonder E, Luger K, Roeder RG, Logie C, Berger SL, Stunnenberg HG: A feed-forward repression mechanism anchors the Sin3/ histone deacetylase and $\mathrm{N}-\mathrm{CoR} / \mathrm{SMRT}$ corepressors on chromatin. Mol Cell Biol 2006, 26:5226-5236

26. Kurdistani SK, Grunstein M: Histone acetylation and deacetylation in yeast. Nat Rev Mol Cell Biol 2003, 4:276-284

27. Hassig CA, Tong JK, Fleischer TC, Owa T, Grable PG, Ayer DE, Schreiber SL: A role for histone deacetylase activity in HDAC1mediated transcriptional repression. Proc Natl Acad Sci USA 1998, 95:3519-3524

28. Yang XJ, Gregoire S: Class II histone deacetylases: from sequence to function, regulation, and clinical implication. Mol Cell Biol 2005, 25:2873-2884

29. Wolffe AP: Histone deacetylase: a regulator of transcription. Science 1996, 272:371-372

30. de Ruijter AJ, van Gennip AH, Caron HN, Kemp S, van Kuilenburg AB: Histone deacetylases (HDACs): characterization of the classical HDAC family. Biochem J 2003, 370:737-749

31. Friedman SL, Rockey DC, McGuire RF, Maher JJ, Boyles JK, Yamasaki G: Isolated hepatic lipocytes and Kupffer cells from normal human liver: morphological and functional characteristics in primary culture. Hepatology 1992, 15:234-243 
32. Tsukamoto H, Cheng S, Blaner WS: Effects of dietary polyunsaturated fat on ethanol-induced Ito cell activation. Am J Physiol 1996, 270:G581-G586

33. Dahl JA, Collas P: A rapid micro chromatin immunoprecipitation assay (microChIP). Nat Protoc 2008, 3:1032-1045

34. Arthur MJ: Collagenases and liver fibrosis. J Hepatol 1995, 22:43-48

35. Han YP, Yan C, Zhou L, Qin L, Tsukamoto H: A matrix metalloproteinase- 9 activation cascade by hepatic stellate cells in trans-differentiation in the three-dimensional extracellular matrix. J Biol Chem 2007, 282:12928-12939

36. Sato H, Kita M, Seiki M: v-Src activates the expression of 92-kDa type IV collagenase gene through the AP-1 site and the GT box homologous to retinoblastoma control elements. A mechanism regulating gene expression independent of that by inflammatory cytokines. J Biol Chem 1993, 268:23460-23468

37. Takahra T, Smart DE, Oakley F, Mann DA: Induction of myofibroblast MMP-9 transcription in three-dimensional collagen I gel cultures: regulation by NF-kappaB. AP-1 and Sp1. Int J Biochem Cell Biol 2004, 36:353-363

38. Tanimura S, Asato K, Fujishiro SH, Kohno M: Specific blockade of the ERK pathway inhibits the invasiveness of tumor cells: down-regulation of matrix metalloproteinase-3/-9/-14 and CD44. Biochem Biophys Res Commun 2003, 304:801-806

39. Raymond L, Eck S, Mollmark J, Hays E, Tomek I, Kantor S, Elliott S, Vincenti M: Interleukin-1 beta induction of matrix metalloproteinase-1 transcription in chondrocytes requires ERK-dependent activation of CCAAT enhancer-binding protein-beta. J Cell Physiol 2006, 207:683-688

40. Yan C, Boyd DD: Regulation of matrix metalloproteinase gene expression. J Cell Physiol 2007, 211:19-26

41. Nelson DE, Ihekwaba AE, Elliott M, Johnson JR, Gibney CA, Foreman BE, Nelson G, See V, Horton CA, Spiller DG, Edwards SW, McDowell HP, Unitt JF, Sullivan E, Grimley R, Benson N, Broomhead D, Kell DB, White MR: Oscillations in NF-kappaB signaling control the dynamics of gene expression. Science 2004, 306:704-708

42. Komarnitsky P, Cho EJ, Buratowski S: Different phosphorylated forms of RNA polymerase II and associated mRNA processing factors during transcription. Genes Dev 2000, 14:2452-2460

43. Birkedal-Hansen H, Moore WG, Bodden MK, Windsor LJ, BirkedalHansen B, DeCarlo A, Engler JA: Matrix metalloproteinases: a review. Crit Rev Oral Biol Med 1993, 4:197-250

44. Page-McCaw A, Ewald AJ, Werb Z: Matrix metalloproteinases and the regulation of tissue remodelling. Nat Rev Mol Cell Biol 2007, 8:221-233

45. Wysocki AB, Staiano-Coico L, Grinnell F: Wound fluid from chronic leg ulcers contains elevated levels of metalloproteinases MMP-2 and MMP-9. J Invest Dermatol 1993, 101:64-68

46. Friedman SL, Roll FJ, Boyles J, Bissell DM: Hepatic lipocytes: the principal collagen-producing cells of normal rat liver. Proc Natl Acad Sci USA 1985, 82:8681-8685

47. Arthur MJ, Friedman SL, Roll FJ, Bissell DM: Lipocytes from normal rat liver release a neutral metalloproteinase that degrades basement membrane (type IV) collagen. J Clin Invest 1989, 84:1076-1085

48. Mutskov V, Felsenfeld G: Silencing of transgene transcription precedes methylation of promoter DNA and histone $\mathrm{H} 3$ lysine 9. EMBO J 2004, 23:138-149

49. Hassig CA, Schreiber SL: Nuclear histone acetylases and deacetylases and transcriptional regulation: HATs off to HDACs. Curr Opin Chem Biol 1997, 1:300-308

50. Heinzel T, Lavinsky RM, Mullen TM, Soderstrom M, Laherty CD, Torchia J, Yang WM, Brard G, Ngo SD, Davie JR, Seto E, Eisenman RN, Rose DW, Glass CK, Rosenfeld MG: A complex containing $\mathrm{N}-\mathrm{CoR}, \mathrm{mSin} 3$ and histone deacetylase mediates transcriptional repression. Nature 1997, 387:43-48

51. Razin A: CpG methylation, chromatin structure and gene silencing-a three-way connection. EMBO J 1998, 17:4905-4908

52. El-Osta A, Wolffe AP: DNA methylation and histone deacetylation in the control of gene expression: basic biochemistry to human development and disease. Gene Expr 2000, 9:63-75

53. Mann J, Oakley F, Akiboye F, Elsharkawy A, Thorne AW, Mann DA Regulation of myofibroblast transdifferentiation by DNA methylation and MeCP2: implications for wound healing and fibrogenesis. Cell Death Differ 2007, 14:275-285

54. Jin C, Li H, Murata T, Sun K, Horikoshi M, Chiu R, Yokoyama KK JDP2, a repressor of AP-1, recruits a histone deacetylase 3 complex to inhibit the retinoic acid-induced differentiation of F9 cells. Mol Cell Biol 2002, 22:4815-4826

55. Gu W, Roeder RG: Activation of p53 sequence-specific DNA binding by acetylation of the p53 C-terminal domain. Cell 1997, 90:595-606

56. Boyes J, Byfield P, Nakatani Y, Ogryzko V: Regulation of activity of the transcription factor GATA-1 by acetylation. Nature 1998, 396:594-598

57. Chen LF, Mu Y, Greene WC: Acetylation of RelA at discrete sites regulates distinct nuclear functions of NF-kappaB. EMBO J 2002, 21:6539-6548

58. Knittel T, Mehde M, Kobold D, Saile B, Dinter C, Ramadori G: Expression patterns of matrix metalloproteinases and their inhibitors in parenchymal and non-parenchymal cells of rat liver: regulation by TNFalpha and TGF-beta1. J Hepatol 1999, 30:48-60

59. Watanabe T, Niioka M, Ishikawa A, Hozawa S, Arai M, Maruyama K, Okada A, Okazaki I: Dynamic change of cells expressing MMP-2 mRNA and MT1-MMP mRNA in the recovery from liver fibrosis in the rat. J Hepatol 2001, 35:465-473

60. Iredale JP, Benyon RC, Arthur MJ, Ferris WF, Alcolado R, Winwood PJ, Clark N, Murphy G: Tissue inhibitor of metalloproteinase-1 messenger RNA expression is enhanced relative to interstitial collagenase messenger RNA in experimental liver injury and fibrosis. Hepatology 1996, 24:176-184 